\title{
Relationships between the work recovery ratio of indentation and plastic parameters for instrumented spherical indentation
}

\author{
Chang Yu, Rong Yang, Yihui Feng, and Yong Huan, State Key Laboratory of Nonlinear Mechanics (LNM), Institute of Mechanics, Chinese Academy \\ of Sciences, Beijing 100190, China \\ Guangjian Peng and Taihua Zhang, College of Mechanical Engineering, Zhejiang University of Technology, Hangzhou 310014, China \\ Address all correspondence to Taihua Zhang at zhangth@zjut.edu.cn
}

(Received 31 October 2014; accepted 23 February 2015)

\begin{abstract}
This paper aims to obtain an analytical expression for the ratio of unloading work of indentation $\left(W_{\mathrm{u}}\right)$ to total loading work of indentation $\left(W_{\mathrm{t}}\right)$ (work recovery ratio of indentation) in instrumented spherical indentation. The expanding cavity model and Lamé solution are used. Three typical stress-strain relations (elastic-perfectly plastic, linear hardening, and power-law hardening) are analyzed. The results of finite-element method coincide with the expressions. The expressions show that the work recovery ratio of indentation is just related to plastic parameters. Furthermore, elastic work $\left(W_{\mathrm{e}}\right)$ are obtained, and it is proved that $W_{\mathrm{e}}$ should be distinguished from $W_{\mathrm{u}}$ in spherical indentation.
\end{abstract}

\section{Introduction}

In recent studies, interest has been intensifying in the development of indentation-based methods to extract material elasticplastic properties. One category of previous studies aimed to obtain the stress-strain curve from a load-depth curve. ${ }^{[1]}$ In another category of studies, many fitting parameters were involved. ${ }^{[2]}$ In this case, it was easy to determine the relationship between the mechanical parameters and the measurement parameters tested. However, these methods led to complex formulations and the parameters. Ogasawara et al. obtained elastic-plastic parameters from measurements at several depths, a process that mimics the dual/plural sharp indentation method. ${ }^{[3]}$ There is a common problem in these methods. That is lack of sufficient theoretical analysis. In our previous work, ${ }^{[4]}$ it was found that a stable solution can be obtained by the use of work recovery ratio of indentation $\left(W_{\mathrm{u}} / W_{\mathrm{t}}\right)$. It seemed to be correlative only with plastic parameters in the power-law hardening material by instrumented spherical indentation. To improve that assumption, and enlarge the scope of the assumption, three typical stress-strain relations are considered to obtain an analytical expression for work recovery ratio of indentation.

A general stress-strain relation for the material can be written as

$$
\tilde{\sigma}= \begin{cases}E \tilde{\varepsilon} & \left(\tilde{\varepsilon}<\varepsilon_{\mathrm{y}}\right) \\ f(\tilde{\varepsilon}) & \left(\tilde{\varepsilon} \geq \varepsilon_{\mathrm{y}}\right)\end{cases}
$$

where $\tilde{\sigma}$ and $\tilde{\varepsilon}$ are the equivalent stress and strain, respectively, $E$ is the elastic modulus, and $\varepsilon_{\mathrm{y}}$ is the yield strain.
The function $f(\tilde{\varepsilon})$ is the constitutive equation of material, which for elastic-perfectly plastic materials takes the form,

$$
\tilde{\sigma}=E \varepsilon_{\mathrm{y}}
$$

while for linear hardening materials, it can be written as

$$
\tilde{\sigma}=E_{\mathrm{p}} \tilde{\varepsilon}+\left(E-E_{\mathrm{p}}\right) \varepsilon_{\mathrm{y}},
$$

and for power-law hardening materials, it can be written as

$$
\tilde{\sigma}=E \varepsilon_{\mathrm{y}}^{1-n} \tilde{\varepsilon}^{n}
$$

where $E_{\mathrm{p}}$ is the hardening modulus, and $n$ is the hardening exponent. These three forms of stress-strain relation are shown in Fig. 1. It should be pointed out that elastic-perfectly plastic materials is included in linear hardening materials as a special case $\left(E_{\mathrm{p}}=0\right)$, but it always be considered as a classical constitutive form. So they are discussed as three separate forms in this paper.

The problem can be simplified by the assumptions given in Johnson's expanding cavity model (ECM) ${ }^{[5]}$ as shown in Fig. 2 $(a)^{[4]}:$ (1) the displacement field produced by the indenter is approximately spherically symmetric; (2) the material under the indenter can be divided into a core zone $(r<a)$, a plastic zone $(a<r<c)$, and an elastic zone $(r>c)$; (3) the material of core zone is assumed to be an incompressible fluid. To obtain a better result of the total loading work of indentation, Johnson's assumption of an incompressible fluid is replaced with an incompressible solid for the core zone by Yang et al. ${ }^{[6]}$ Meanwhile, the state of the material of core zone is 

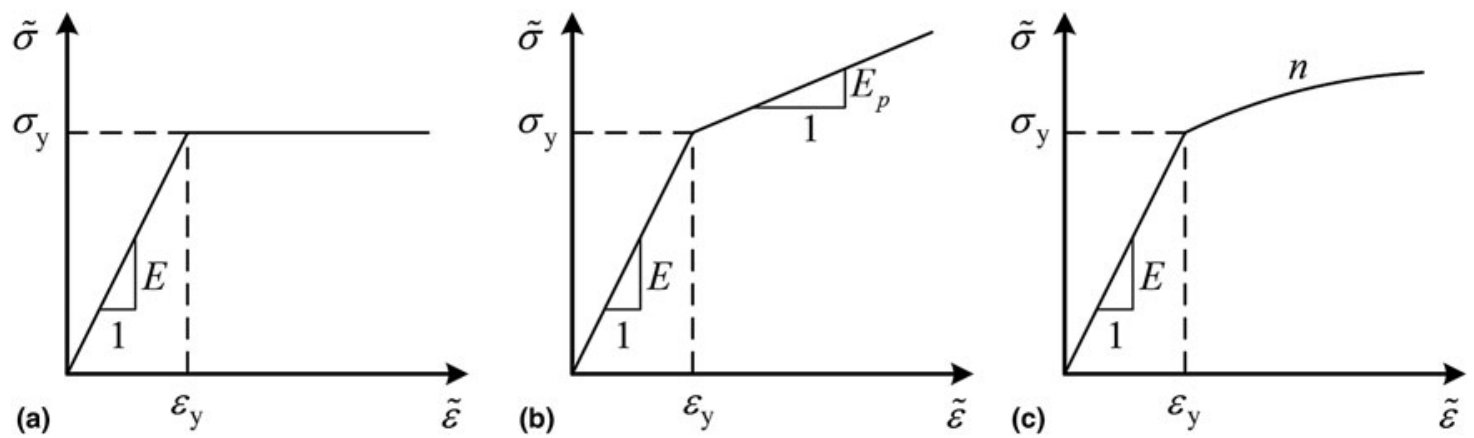

Figure 1. Curves of three typical stress-strain relations. (a) Elastic-perfectly plastic, (b) linear hardening, and (c) power-law hardening.

considered uniform with the same value as that at $r=a$, i.e., on the inner boundary of the plastic zone.

In fact, interface friction has influence on $W_{\mathrm{t}}$ and $W_{\mathrm{u}}$, but it will make the problem too perplexed to obtain analytical solutions and can be corrected by the finite-element method (FEM). Therefore, interface friction is neglected in this paper.

\section{Theoretical analysis}

Based on the spherically symmetric assumption in ECM, seven unknown parameters are considered: $u_{r}, \varepsilon_{r}, \varepsilon_{\theta}, \varepsilon_{\varphi}, \sigma_{r}, \sigma_{\theta}$, and $\sigma_{\varphi}$. Relationships about these parameters are:

the geometric equations

$$
\left\{\begin{array}{l}
\varepsilon_{r}=\frac{d u_{r}}{d r} \\
\varepsilon_{\theta}=\varepsilon_{\varphi}=\frac{u_{r}}{r}
\end{array}\right.
$$

and the balance equation

$$
\frac{d \sigma_{r}}{d r}+2 \frac{\sigma_{r}-\sigma_{\theta}}{r}=0
$$

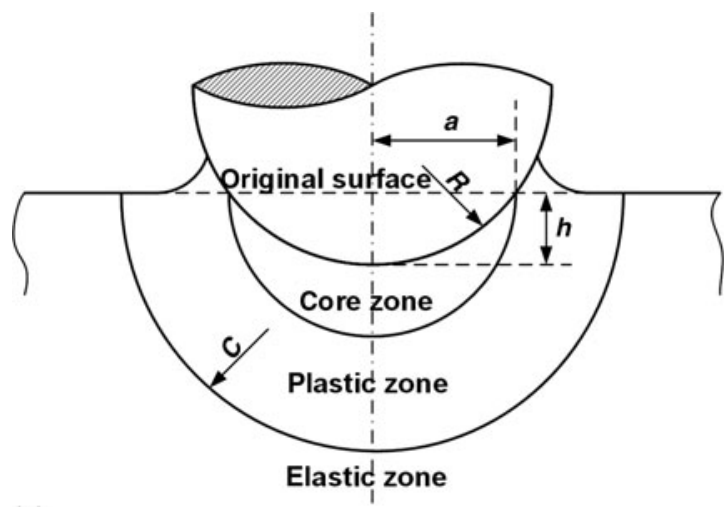

(a)
The equivalent stress and $\operatorname{strain}^{[7]}$ are

$$
\begin{aligned}
\tilde{\sigma} & =\frac{1}{\sqrt{2}} \sqrt{\left(\sigma_{r}-\sigma_{\theta}\right)^{2}+\left(\sigma_{\theta}-\sigma_{\varphi}\right)^{2}+\left(\sigma_{\varphi}-\sigma_{r}\right)^{2}} \\
& =\sigma_{\theta}-\sigma_{r}, \\
\tilde{\varepsilon} & =\frac{\sqrt{2}}{3} \sqrt{\left(\varepsilon_{r}-\varepsilon_{\theta}\right)^{2}+\left(\varepsilon_{\theta}-\varepsilon_{\varphi}\right)^{2}+\left(\varepsilon_{\varphi}-\varepsilon_{r}\right)^{2}} \\
& =\frac{2}{3}\left(\varepsilon_{\theta}-\varepsilon_{r}\right) .
\end{aligned}
$$

By assuming material incompressibility (specifically the Poisson ratio of $v=0.5$ ), Gao and Jing, ${ }^{[8]}$ radial displacement is adaptable to the volume of part of the indenter which is under the surface of sample. It can be written as

$$
V=\frac{2}{3} \pi\left[\left(r+u_{r}\right)^{3}-r^{3}\right]=\frac{2}{3} \pi u_{r}\left(3 r^{2}+3 r u_{r}+u_{r}^{2}\right) .
$$

It is easy to obtain $u_{r} / r=\sqrt[3]{3 V /\left(2 \pi r^{3}\right)+1}-1$. By considering the ratio of depth $(h / R)$ as a constant $0.3,{ }^{[9]} u_{r} / r$ is not larger than 0.1 and decreases rapidly. It means that a small

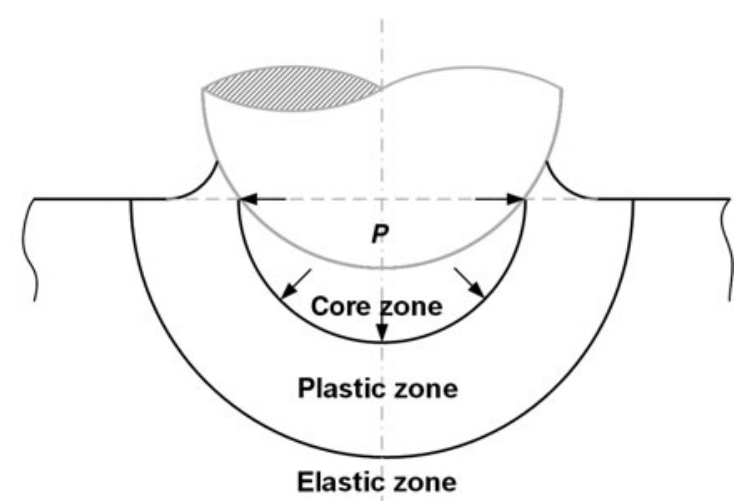

(b)

Figure 2. ECM in which the deformation area below the indenter is divided into three parts. The effect of pile-up or sink-in is equivalent to some fraction of hydrostatic core volume displaced by indenter. (a) The process of loading, which is equivalent to (b) the stress $p$ acting on the edge of the spherical cavity. ${ }^{[4]}$ 
deformation assumption $\left(u_{r} \ll r\right)$ is reasonable and Eq. (9) can be simplified into

$$
V=2 \pi u_{r} r^{2}
$$

and $u_{r}$ can be written as

$$
u_{r}=\frac{V}{2 \pi r^{2}} .
$$

Substituting Eq. (11) into (5) yields

$$
\left\{\begin{array}{l}
\varepsilon_{r}=-\frac{V}{\pi r^{3}} \\
\varepsilon_{\theta}=\varepsilon_{\varphi}=\frac{V}{2 \pi r^{3}} .
\end{array}\right.
$$

By substituting Eqs. (12) into (8), the equivalent strain can be written as

$$
\tilde{\varepsilon}=\frac{V}{\pi r^{3}}
$$

At the outer boundary of plastic zone

$$
\left.\tilde{\varepsilon}\right|_{r=c}=\frac{V}{\pi c^{3}}=\varepsilon_{\mathrm{y}}
$$

By substituting Eq. (14) into (13), the relationship between equivalent strain and radius is

$$
\tilde{\varepsilon}=\frac{c^{3}}{r^{3}} \varepsilon_{\mathrm{y}}
$$

\section{Total loading work of indentation and elastic work of indentation}

The energy density can be written as

$$
w_{\mathrm{t}}=\int \tilde{\sigma} d \tilde{\varepsilon}
$$

Inside the elastic zone $(r>c)$, substituting equivalent stress Eq. (1) into (16) yields

$$
w_{\mathrm{t}}^{\mathrm{e}}=\int \tilde{\sigma} d \tilde{\varepsilon}=\int E \tilde{\varepsilon} d \tilde{\varepsilon}=\frac{1}{2} E \tilde{\varepsilon}^{2}+C_{1} .
$$

By considering the boundary condition $\left.w_{\mathrm{t}}^{\mathrm{e}}\right|_{\tilde{\varepsilon}=0}=C_{1}=0$, we get

$$
w_{\mathrm{t}}^{\mathrm{e}}=\frac{1}{2} E \tilde{\varepsilon}^{2} .
$$

Inside the plastic zone $(a<r<c)$, substituting equivalent stress Eqs. (2)-(4) into (16), and considering boundary condition $\left.w_{\mathrm{t}}^{\mathrm{p}}\right|_{\tilde{\varepsilon}=\varepsilon_{\mathrm{y}}}=\left.w_{\mathrm{t}}^{\mathrm{e}}\right|_{\tilde{\varepsilon}=\varepsilon_{\mathrm{y}}}$, three different forms of the energy density with different stress-strain relations can be written as

$$
\left\{\begin{array}{l}
w_{\mathrm{t}}^{\mathrm{p}}=E \varepsilon_{\mathrm{y}} \tilde{\varepsilon}-\frac{1}{2} E \varepsilon_{\mathrm{y}}^{2} \\
w_{\mathrm{t}}^{\mathrm{p}}=\frac{1}{2} E_{\mathrm{p}} \tilde{\varepsilon}^{2}+\left(E-E_{\mathrm{p}}\right) \varepsilon_{\mathrm{y}} \tilde{\varepsilon}-\frac{1}{2}\left(E-E_{\mathrm{p}}\right) \varepsilon_{\mathrm{y}}^{2} \\
w_{\mathrm{t}}^{\mathrm{p}}=\frac{1}{n+1} E \varepsilon_{\mathrm{y}}^{1-n} \tilde{\varepsilon}^{n+1}+\frac{n-1}{2(n+1)} E \varepsilon_{\mathrm{y}}^{2}
\end{array}\right.
$$

Inside the core zone $(r<a)$, due to the incompressible assumption, the equivalent stress and strain are equal to the edge of core zone $(r=a)$. Substituting the radius into Eq. (15),

$$
\tilde{\varepsilon}=\left.\tilde{\varepsilon}\right|_{r=a}=\frac{c^{3}}{a^{3}} \varepsilon_{\mathrm{y}}
$$

Substituting Eq. (20) into (2)-(4), we can get the equivalent stress. Then substituting equivalent stress and strain into Eq. (16), three different forms of the energy density within different stress-strain relations can be written as

$$
\left\{\begin{array}{l}
w_{\mathrm{t}}^{c}=E \varepsilon_{\mathrm{y}}^{2} \frac{c^{3}}{a^{3}} \\
w_{\mathrm{t}}^{c}=E \varepsilon_{\mathrm{y}}^{2}\left[\frac{E_{\mathrm{p}}}{E} \frac{c^{6}}{a^{6}}+\left(1-\frac{E_{\mathrm{p}}}{E}\right) \frac{c^{3}}{a^{3}}\right] \\
w_{\mathrm{t}}^{c}=E \varepsilon_{\mathrm{y}}^{2} \frac{c^{3(n+1)}}{a^{3(n+1)}}
\end{array}\right.
$$

The total loading work of indentation can be determined using

$$
W_{\mathrm{t}}=\int_{c}^{\infty} 2 \pi r^{2} w_{\mathrm{t}}^{\mathrm{e}} d r+\int_{a}^{c} 2 \pi r^{2} w_{\mathrm{t}}^{\mathrm{p}} d r+w_{\mathrm{t}}^{c} V_{c}
$$

where $V_{c}$ is the volume of core zone [Fig. 2(a)], which can be determined by

$$
V_{c}=\frac{2}{3} \pi a^{3}-V=\frac{2}{3} \pi a^{3}-\pi c^{3} \varepsilon_{\mathrm{y}} .
$$

Substituting Eqs. (15), (18), (19), (21), and (23) into (22), the analytical expression of total loading work of indentation is

$$
\left\{\begin{array}{l}
W_{t}=\pi E \varepsilon_{y}^{2}\left[\frac{1}{3} a^{3}+2 c^{3} \ln \frac{c}{a}+\frac{c^{3}}{a^{3}}\left(\frac{2}{3} a^{3}-c^{3} \varepsilon_{y}\right)\right] \\
W_{t}=\pi E \varepsilon_{y}^{2}\left\{\frac{1}{3} \frac{E_{p}}{E} \frac{c^{6}}{a^{3}}+\left(1-\frac{E_{p}}{E}\right)\left(\frac{1}{3} a^{3}+2 c^{3} \ln \frac{c}{a}\right)+\right. \\
\left.\left[\frac{E_{p}}{E} \frac{c^{6}}{a^{6}}+\left(1-\frac{E_{p}}{E}\right) \frac{c^{3}}{a^{3}}\right]\left(\frac{2}{3} a^{3}-c^{3} \varepsilon_{y}\right)\right\} \\
W_{t}=\pi E \varepsilon_{y}^{2}\left[\frac{2 n c^{3}-(n-1) a^{3}}{3(n+1)}+\frac{2 c^{3}}{3 n(n+1)}\left(\frac{c^{3 n}}{a^{3 n}}-1\right)+\right. \\
\left.\frac{c^{3(n+1)}}{a^{3(n+1)}}\left(\frac{2}{3} a^{3}-c^{3} \varepsilon_{y}\right)\right]
\end{array}\right.
$$


The elastic work can be determined by

$$
W_{\mathrm{e}}=\int_{a}^{\infty} 2 \pi r^{2} w_{\mathrm{t}}^{\mathrm{e}} d r=3 \pi E \varepsilon_{\mathrm{y}}^{2} \frac{c^{6}}{a^{3}} .
$$

\section{Unloading work of indentation}

During unloading, little plastic deformation occurs in the material being tested, and this behavior has been confirmed by finiteelement analysis. ${ }^{[10]}$ Based on this behavior, we assume that only elastic deformation occurs during unloading. According to the assumption of spherically symmetric strain distribution in Johnson's expanding cavity model, the stress $p$ acting at the edge of the spherical cavity [Fig. 2(b)] is reduced to zero during unloading. This behavior can be equivalently replaced by adding to the elastic-plastic stress field at the end of loading. The elastic stress field can be described by Lamé's solution ${ }^{[7]}$

$$
\tilde{\sigma}=\frac{3}{2} \frac{a^{3}}{r^{3}} p .
$$

The energy density can be written as

$$
w_{\mathrm{u}}=\int \tilde{\sigma} d \tilde{\varepsilon}=\int \tilde{\sigma} d \frac{\tilde{\sigma}}{E}=\frac{1}{2 E} \tilde{\sigma}^{2} .
$$

Substituting Eq. (26) into (27) yields

$$
w_{\mathrm{u}}=\frac{9}{8 E} \frac{a^{6}}{r^{6}} p^{2}
$$

and the unloading work of indentation can be written as

$$
\begin{aligned}
W_{\mathrm{u}} & =\int_{a}^{\infty} 2 \pi r^{2} w_{\mathrm{u}} d r=\int_{a}^{\infty} 2 \pi r^{2}\left(\frac{9}{8 E} \frac{a^{6}}{r^{6}} p^{2}\right) d r \\
& =\frac{3}{4} \frac{\pi}{E} a^{3} p^{2}
\end{aligned}
$$

where $p=-\sigma_{r} / r=a$ comes from the end of loading.

Substituting Eqs. (2)-(4) into (6), three different forms of $\sigma_{r}$ within different stress-strain relations can be obtained as

$$
\left\{\begin{array}{l}
\sigma_{r}=2 E \varepsilon_{\mathrm{y}} \ln \frac{r}{c}-\frac{2}{3} E \varepsilon_{\mathrm{y}} \\
\sigma_{r}=-\frac{2}{3} E_{\mathrm{p}} \varepsilon_{\mathrm{y}} \frac{c^{3}}{r^{3}}-\frac{2}{3}\left(E-E_{\mathrm{p}}\right) \varepsilon_{\mathrm{y}}+2\left(E-E_{\mathrm{p}}\right) \varepsilon_{\mathrm{y}} \ln \frac{r}{c} \\
\sigma_{r}=-\frac{2}{3 n} E \varepsilon_{\mathrm{y}} \frac{c^{3 n}}{r^{3 n}}+\frac{2(1-n)}{3 n} E \varepsilon_{\mathrm{y}}
\end{array}\right.
$$

At $r=a, p$ can be written as

$$
\left\{\begin{array}{l}
p=\frac{2}{3} E \varepsilon_{\mathrm{y}}-2 E \varepsilon_{\mathrm{y}} \ln \frac{a}{c} \\
p=\frac{2}{3} E_{\mathrm{p}} \varepsilon_{\mathrm{y}} \frac{c^{3}}{a^{3}}+\frac{2}{3}\left(E-E_{\mathrm{p}}\right) \varepsilon_{\mathrm{y}}-2\left(E-E_{\mathrm{p}}\right) \varepsilon_{\mathrm{y}} \ln \frac{a}{c} \\
p=\frac{2}{3 n} E \varepsilon_{\mathrm{y}} \frac{c^{3 n}}{a^{3 n}}+\frac{2(n-1)}{3 n} E \varepsilon_{\mathrm{y}}
\end{array}\right.
$$

Substituting Eqs. (31) into (29) yields

$$
\left\{\begin{array}{l}
W_{\mathrm{u}}=3 \pi E \varepsilon_{\mathrm{y}}^{2} a^{3}\left(\frac{1}{3}-\ln \frac{a}{c}\right)^{2} \\
W_{\mathrm{u}}=3 \pi E \varepsilon_{\mathrm{y}}^{2} a^{3}\left[\frac{1}{3} \frac{E_{\mathrm{p}}}{E} \frac{c^{3}}{a^{3}}+\left(1-\frac{E_{\mathrm{p}}}{E}\right)\left(\frac{1}{3}-\ln \frac{a}{c}\right)\right]^{2} \\
W_{\mathrm{u}}=\frac{1}{3 n^{2}} \pi E \varepsilon_{\mathrm{y}}^{2} a^{3}\left(\frac{c^{3 n}}{a^{3 n}}+n-1\right)^{2}
\end{array}\right.
$$

Comparing Eqs. (25) and (32) can result in that $W_{\mathrm{e}}$ should be distinguished from $W_{\mathrm{u}}$, and their equivalence suggested in ISO14577 ${ }^{[11]}$ is not proper. An analogous conclusion has been obtained by Yang et al. in conical indentation. ${ }^{[12]}$

\section{Work recovery ratio of indentation}

By combining Eqs. (32) and (24) and simplifying, work recovery ratio of indentation can be written for elastic-perfectly plastic, as

$$
\frac{W_{u}}{W_{t}}=\frac{[1-3 \ln (a / c)]^{2}}{1+\left(c^{3} / a^{3}\right)\left[2+6 \ln (a / c)-3\left(c^{3} / a^{3}\right) \varepsilon_{y}\right]}
$$

and for linear hardening, as

$$
\begin{aligned}
\frac{W_{u}}{W_{t}}= & \frac{\left\{\left(E_{p} / E\right)\left(c^{3} / a^{3}\right)+\left(1-E_{p} / E\right)[1-3 \ln (a / c)]\right\}^{2}}{3\left(E_{p} / E\right)\left(c^{6} / a^{6}\right)\left(1-c^{3} \varepsilon_{y} / a^{3}\right)+} \\
& \left(1-E_{p} / E\right)\left\{1+\left(c^{3} / a^{3}\right)\left[2+6 \ln (a / c)-3 c^{3} \varepsilon_{y} / a^{3}\right]\right\}
\end{aligned}
$$

and for power-law hardening, as

$$
\begin{aligned}
\frac{W_{u}}{W_{t}}= & \frac{\left(c^{3 n} / a^{3 n}+n-1\right)^{2} / n^{2}}{\left[2 n c^{3} / a^{3}-(n-1)\right] /(n+1)+(c / a)^{3(n+1)}\left(2-3 c^{3} \varepsilon_{y} / a^{3}\right)+} \\
& 2\left(c^{3} / a^{3}\right)\left(c^{3 n} / a^{3 n}-1\right) /[n(n+1)]
\end{aligned}
$$

when $E_{\mathrm{p}}=0$ Eq. (34) will equal to Eq. (33), it means that elastic-perfectly plastic materials is included in linear hardening materials as a special case.

Equations (33)-(35) show that work recovery ratio of indentation is related to plastic parameters $\left(\varepsilon_{\mathrm{y}}, E_{\mathrm{p}} / E\right.$, or $\left.n\right)$ and geometrical parameters $(a, c)$. Based on the geometrical relationship [see Fig. 2(a)], we can obtain

$$
\begin{gathered}
a=\sqrt{2 R h-h^{2}}, \\
V=\frac{1}{3} \pi h^{2}(3 R-h),
\end{gathered}
$$

where $R$ is the radius of indenter, and $h$ is the indentation depth.

Substituting Eqs. (37) into (14), $c$ is determined by

$$
c=\sqrt[3]{\frac{h^{2}(3 R-h)}{3 \varepsilon_{\mathrm{y}}}},
$$


and $c / a$ can be written as

$$
\frac{c}{a}=\frac{\sqrt[3]{h^{2}(3 R-h) /\left(3 \varepsilon_{y}\right)}}{\sqrt{2 R h-h^{2}}}=\frac{\sqrt[3]{(h / R)^{2}(3-h / R) /\left(3 \varepsilon_{y}\right)}}{\sqrt{2(h / R)-(h / R)^{2}}}
$$

By considering the ratio of depth $(h / R)$ as a constant $0.3,{ }^{[9]} c / a$ can be simplified as

$$
\frac{c}{a}=0.606 \varepsilon_{\mathrm{y}}^{-(1 / 3)}
$$

Substituting Eqs. (40) into (33)-(35) yields, the simplified work recovery ratio can be written for elastic-perfectly plastic, as

$$
\frac{W_{\mathrm{u}}}{W_{\mathrm{t}}}=\frac{\varepsilon_{\mathrm{y}}\left(0.503+\ln \varepsilon_{\mathrm{y}}\right)^{2}}{\varepsilon_{\mathrm{y}}-0.372-0.445 \ln \varepsilon_{\mathrm{y}}},
$$

and for linear hardening, as

$$
\frac{W_{u}}{W_{t}}=\frac{\left[0.223 E_{p} / E-\left(1-E_{p} / E\right)\left(0.503 \varepsilon_{y}+\varepsilon_{y} \ln \varepsilon_{y}\right)\right]^{2}}{0.117 E_{p} / E+\left(1-E_{p} / E\right)\left(\varepsilon_{y}^{2}-0.372 \varepsilon_{y}-0.445 \varepsilon_{y} \ln \varepsilon_{y}\right)}
$$

and for power-law hardening, as

$$
\begin{aligned}
\frac{W_{\mathrm{u}}}{W_{\mathrm{t}}}=\frac{(n+1) \varepsilon_{\mathrm{y}}^{n+1}\left(0.223^{n} \varepsilon_{\mathrm{y}}^{-n}+n-1\right)^{2}}{-n^{2}(n-1) \varepsilon_{\mathrm{y}}^{n+1}+0.446 n\left(n^{2}-1\right) \varepsilon_{\mathrm{y}}^{n}+} & .2 \times 0.223^{n+1}\left(n^{3}+n^{2}+n\right)-3 \times 0.223^{n+2} n^{2}(n+1)
\end{aligned}
$$

Equations (41)-(43) show that work recovery ratio of indentation is related to plastic parameters $\left(\varepsilon_{\mathrm{y}}, E_{\mathrm{p}} / E\right.$ or $\left.n\right)$. The plastic parameters can be obtained by solving the work recovery ratio of indentation and Meyer's coefficient simultaneously. ${ }^{[4,9]}$

\section{Finite-element method}

FEM is used to determine the accuracy of the analytical expressions. Plastic parameters used in the FEM calculations cover most of metal materials. Elastic modulus $(E)$ is 210 $\mathrm{GPa}$, because numerical simulations are dimensionless and the effect of the elastic modulus is linear. To make these simulations consistent with reality, we chose to approximate the elastic modulus of steel using the value of $210 \mathrm{GPa}$. Poisson's ratio $(v)$ is 0.3 . Different plastic parameters chosen cover the following range: $\varepsilon_{\mathrm{y}}$ of $10^{-3}-10^{-2}, E_{\mathrm{p}} / E$ of $0.02-$ 0.10 (for linear hardening) and $n$ of $0.1-0.5$ (for power-law hardening), which encompass most engineering metals. Interface friction is neglected to match the assumption in theoretical analysis.

The detail parameters and results are shown in Fig. 3. The curves are analytical results from Eqs. (41)-(43), and the points are FEM results. It can be found that they coincide well. Figure 3 shows that there is a relationship of quadratic function
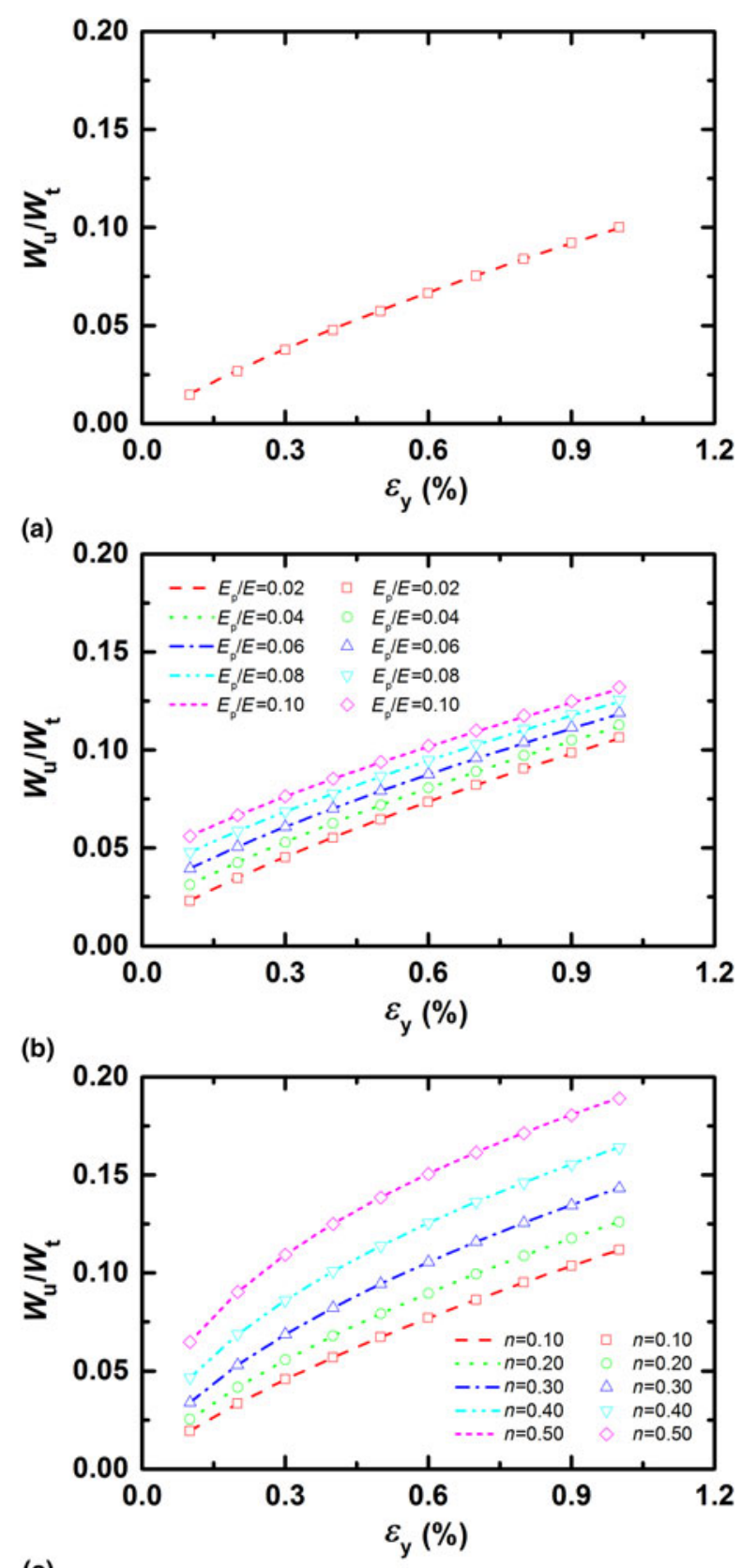

(c)

Figure 3. Analytical results (curves) and FEM results (points) of work recovery ratio of indentation for three stress-strain relations. (a)

Elastic-perfectly plastic, (b) linear hardening, and (c) power-law hardening.

between the work recovery ratio and the yield strain. By fitting the parameters, the more simplified work recovery ratio can be written for elastic-perfectly plastic, as

$$
\frac{W_{\mathrm{u}}}{W_{\mathrm{t}}}=-241.73 \varepsilon_{\mathrm{y}}^{2}+11.962 \varepsilon_{\mathrm{y}}+0.0040
$$

and for linear hardening, as 


$$
\begin{aligned}
\frac{W_{\mathrm{u}}}{W_{\mathrm{t}}}= & {\left[-1002.2\left(\frac{E_{\mathrm{p}}}{E}\right)^{2}+444.85\left(\frac{E_{\mathrm{p}}}{E}\right)-241.73\right] \varepsilon_{\mathrm{y}}^{2} } \\
& +\left[14.201\left(\frac{E_{\mathrm{p}}}{E}\right)^{2}-15.923\left(\frac{E_{\mathrm{p}}}{E}\right)+11.962\right] \varepsilon_{\mathrm{y}} \\
& +\left[0.0532\left(\frac{E_{\mathrm{p}}}{E}\right)^{2}+0.4183\left(\frac{E_{\mathrm{p}}}{E}\right)+0.0040\right],
\end{aligned}
$$

and for power-law hardening, as

$$
\begin{aligned}
\frac{W_{\mathrm{u}}}{W_{\mathrm{t}}}= & \left(-915.51 n^{2}-823.37 n-237.46\right) \varepsilon_{\mathrm{y}}^{2} \\
& +\left(7.0211 n^{2}+18.843 n+11.790\right) \varepsilon_{\mathrm{y}} \\
& +\left(0.1891 n^{2}-0.0170 n+0.0064\right) .
\end{aligned}
$$

8. X.L. Gao and X.N. Jing: Two new expanding cavity models for indentation deformations of elastic strain-hardening materials. Int. J. Solids Struct. 43, 2193 (2006).

9. P. Jiang, T.H. Zhang, Y.H. Feng, R. Yang, and N.G. Liang: Determination of plastic properties by instrumented spherical indentation: expanding cavity model and similarity solution approach. J. Mater. Res. 24, 1045 (2009).

10. W.C. Oliver and G.M. Pharr: Measurement of hardness and elastic modulus by instrumented indentation advances in understanding and refinement. J. Mater. Res. 19, 3 (2004).

11. ISO 14577:2002, Metallic materials-instrumented indentation test for hardness and materials parameters.

12. R. Yang, T.H. Zhang, P. Jiang, and Y.L. Bai: Experimental verification and theoretical analysis of the relationships between hardness, elastic modulus, and the work of indentation. Appl. Phys. Lett. 92, 231906 (2008).

\section{Conclusion}

In this paper, analytical expressions for the work recovery ratio of indentation for three typical stress-strain relations are obtained. It is proved that the work recovery ratio of indentation is just related to plastic parameters, and there is positive correlation between the work recovery ratio of indentation and plastic parameters. It is also proved that $W_{\mathrm{e}}$ should be distinguished from $W_{\mathrm{u}}$ in spherical indentation as well as that is in conical indentation. It provides us with a method to estimate the ability of plastic deformation of an unknown material by instrumented spherical indentation. Furthermore, by solving the work recovery ratio of indentation and Meyer's coefficient simultaneously, the plastic parameters can be obtained exactly.

\section{Acknowledgments}

Authors gratefully acknowledge the financial support from the National Natural Science Foundation of China (Grant Nos. $11025212,11272318,11172305,11302231,11372323$, and 11402233).

\section{References}

1. A. Hasanov: An inversion method for identification of elastoplastic properties for engineering materials from limited spherical indentation measurements. Inverse Probl. Sci. Eng. 15, 601 (2007).

2. M.Q. Le: Material characterization by instrumented spherical indentation. Mech. Mater. 46, 42 (2012).

3. N. Ogasawara, N. Chiba, and X. Chen: A simple framework of spherical indentation for measuring elastoplastic properties. Mech. Mater. 41, 1025 (2009).

4. C. Yu, Y.H. Feng, R. Yang, G.J. Peng, Z.K. Lu, and T.H. Zhang: An integrated method to determine elastic-plastic parameters by instrumented spherical indentation. J. Mater. Res. 29, 1095 (2014).

5. K.L. Johnson: The correlation of indentation experiments. J. Mech. Phys. Solids 18, 115 (1970).

6. R. Yang, T.H. Zhang, and Y.H. Feng: Theoretical analysis of the relationships between hardness, elastic modulus, and the work of indentation for work-hardening materials. J. Mater. Res. 25, 2072 (2010).

7. R. Hill: The Mathematical Theory of Plasticity (Oxford University Press, New York, 1998) 